\title{
PPAR $\alpha$ activation improves endothelial dysfunction and reduces fibrosis and portal pressure in cirrhotic rats
}

\author{
Aina Rodríguez-Vilarrupla ${ }^{1, *}$, Bàrbara Laviña ${ }^{1}$, Héctor García-Calderó ${ }^{1}$, Lucia Russo ${ }^{1}$, \\ Eugenio Rosado ${ }^{1}$, Núria Roglans ${ }^{2}$, Jaume Bosch ${ }^{1}$, Joan Carles García-Pagán ${ }^{1}$ \\ ${ }^{1}$ Hepatic Hemodynamic Laboratory, Liver Unit, IMDIM, Hospital Clínic, Institut d'Investigacions Biomèdiques August Pi i Sunyer (IDIBAPS) and \\ Ciberehd, University of Barcelona, Spain; ${ }^{2}$ Pharmacology Unit, Department of Pharmacology and Therapeutic Chemistry, \\ School of Pharmacy, University of Barcelona, Spain
}

\begin{abstract}
Background \& Aims: Peroxisome proliferator-activated receptor $\alpha(\mathrm{PPAR} \alpha)$ is a transcription factor activated by ligands that regulates genes related to vascular tone, oxidative stress, and fibrogenesis, pathways implicated in the development of cirrhosis and portal hypertension. This study aims at evaluating the effects of PPAR $\alpha$ activation with fenofibrate on hepatic and systemic hemodynamics, hepatic endothelial dysfunction, and hepatic fibrosis in $\mathrm{CCl}_{4}$-cirrhotic rats.
\end{abstract}

Methods: Mean arterial pressure (MAP), portal pressure (PP), and portal blood flow (PBF) were measured in cirrhotic rats treated with oral fenofibrate $(25 \mathrm{mg} / \mathrm{kg} /$ day, $\mathrm{n}=10)$ or its vehicle $(\mathrm{n}=12)$ for 7 days. The liver was then perfused and dose-relaxation curves to acetylcholine (Ach) were performed. We also evaluated Sirius Red staining of liver sections, collagen-I mRNA expression, and smooth muscle actin ( $\alpha$-SMA) protein expression, cyclo-oxygenase-1 (COX-1) protein expression, and cGMP levels in liver homogenates, and $\mathrm{TXB}_{2}$ production in perfusates. Nitric oxide (NO) bioavailability and eNOS activation were measured in hepatic endothelial cells (HEC) isolated from cirrhotic rat livers.

Results: $\mathrm{CCl}_{4}$ cirrhotic rats treated with fenofibrate had a significantly lower PP (-29\%) and higher MAP than those treated with vehicle. These effects were associated with a significant reduction in hepatic fibrosis and improved vasodilatory response to acetylcholine. Moreover, a reduction in COX-1 expression and $\mathrm{TXB}_{2}$ production in rats receiving fenofibrate and a significant increase in NO bioavailability in HEC with fenofibrate were observed.

Conclusions: PPAR $\alpha$ activation markedly reduced PP and liver fibrosis and improved hepatic endothelial dysfunction in cirrhotic rats, suggesting it may represent a new therapeutic strategy for portal hypertension in cirrhosis.

Keywords: Fenofibrate; Nitric oxide; COX pathway; Portal hypertension. Received 1 August 2011; received in revised form 12 December 2011; accepted 14 December 2011; available online 13 January 2012

* Corresponding author. Address: Hepatic Hemodynamic Laboratory, Liver Unit Hospital Clínic, Villarroel 170, 08036 Barcelona, Spain. Tel.: +34 93227 5400x2971; fax: +34932279856.

E-mail address: arodr@clinic.ub.es (A. Rodríguez-Vilarrupla).

Abbreviations: PPAR $\alpha$, peroxisome proliferator-activated receptor $\alpha ; \mathrm{CCl}_{4}$, carbon tetrachloride; MAP, mean arterial pressure; PP, portal pressure; PBF, portal blood flow; Mtx, methoxamine; $\alpha$-SMA, alfa smooth muscle actin; NO, nitric oxide; TAA, thioacetamide; TG, triglycerides; DAF-FM-DA, 4-amino-5-methylamino-2', 7'-difluorofluorescein diacetate.
(C) 2012 European Association for the Study of the Liver. Published by Elsevier B.V. All rights reserved.

\section{Introduction}

Increased resistance to portal blood flow is the primary factor in the pathophysiology of portal hypertension, the main complication of cirrhosis [1]. Increased resistance to portal blood flow in cirrhotic livers results both from architectural alterations of the liver parenchyma and from a dynamic increase in the hepatic vascular tone. Sinusoidal endothelial cell dysfunction, characterized by impaired endothelium-dependent vasodilation, is an important mechanism of the increased vascular tone of cirrhotic livers and has been attributed, among others, to increased release of cyclo-oxygenase-1 (COX-1)-derived vasoconstrictive prostanoids $[2,3]$ and to reduced bioavailability of the vasodilator nitric oxide (NO) within the liver [4].

Peroxisome proliferator-activated receptor $\alpha(\operatorname{PPAR} \alpha)$ is a member of the nuclear receptor superfamily and acts as a ligand-activated transcription factor. PPAR $\alpha$ is predominantly expressed in the liver [5]. Identification of PPAR target genes first illustrated that PPAR $\alpha$ is a major regulator of fatty acid homeostasis [6], but it is well recognized that the transcriptional program of PPAR $\alpha$ also includes genes involved in the regulation of vascular tone, oxidative stress, and fibrosis $[7,8]$.

PPAR $\alpha$ receptors are activated by natural or synthetic ligands, such as fibrates [9]. Fibrates are first-line drugs in the treatment of hyperlipidemia and have also been shown to improve endothelial dysfunction in arterial hypertension [10], diabetes [11], and ischemia/reperfusion injury [12]. PPAR $\alpha$ is expressed in endothelial cells and it has been demonstrated that PPAR $\alpha$ activation is involved in several endothelial functions as leukocyte recruitment, inflammatory signalling, and vascular functions [13]. The beneficial effects of fenofibrate on vascular function may be partly due to improved endothelial NO availability, as it has been demonstrated that PPAR $\alpha$ activation increases NO production in endothelial cells [14-16]. Besides, PPAR $\alpha$ activation has also been shown to decrease the expression of COX-1 and the release of thromboxane $\mathrm{A}_{2}\left(\mathrm{TXA}_{2}\right)$ [17]. Additionally, it has been observed that PPAR $\alpha$ activation exerts antifibrotic effects on the TAAinduced model of hepatic fibrosis [18]. Altogether these findings

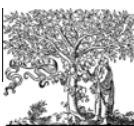

Journal of Hepatology 2012 vol. 56 | 1033-1039 


\section{Research Article}

suggest that activation of PPAR $\alpha$ receptors could have beneficial effects in the pathophysiological mechanisms involved in the development of portal hypertension in cirrhosis.

The aim of this study was to evaluate the effects of PPAR $\alpha$ activation with fenofibrate on hepatic and systemic hemodynamics, hepatic endothelial dysfunction, and hepatic fibrosis in $\mathrm{CCl}_{4-}$ cirrhotic rats.

\section{Materials and methods}

Experimental protocols

Cirrhosis was induced in male Wistar rats (50-75 g) by inhalation of $\mathrm{CCl}_{4}$ three times a week during approximately $12-15$ weeks as previously described [19] When cirrhotic rats had developed ascites, administration of $\mathrm{CCl}_{4}$ and phenobarbital was discontinued. One week later, the animals were distributed randomly into two groups. Each group was fed, respectively, with a control diet or with a diet containing fenofibrate (Sigma, Tres Cantos, Madrid, Spain) to proportionate a daily therapeutic dose of $25 \mathrm{mg} / \mathrm{kg}$ for 7 days (which is in the range previously reported in rats with no toxic effects) [20]. The diets were prepared as previously described [21] by soaking in an acetone solution of the drug. To avoid any possible effect of the solvent, the control diet was also soaked in acetone and dried. The animals were kept in environmentally controlled animal facilities at the Institut d'Investigacions Biomèdiques August Pi i Sunyer (IDIBAPS). All experiments were approved by the Laboratory Animal Care and Use Committee of the University of Barcelona and were conducted in accordance with Guide for the Care and Use of Laboratory Animals (National Institutes of Health, NIH Publication 86-23, revised 1996).

Evaluation of PPAR $\alpha$ activation

\section{PPAR $\alpha$ gene expression}

Expression of PPAR $\alpha$ was analyzed in total RNA from cirrhotic livers using predesigned gene expression assays obtained from Applied Biosystems (Applied Biosystems, Foster City, CA) according to the manufacturer's protocol and reported relative to the endogenous control $18 \mathrm{~S}$. All PCR reactions were performed in duplicate and using nuclease-free water as no template control.

Protein expression of PPAR $\alpha$ and Acyl-Coenzyme A oxydase 1

PPAR $\alpha$ protein expression was determined in hepatic crude nuclear extracts obtained as previously described [22], using a mouse antibody against PPAR $\alpha$ (Abcam, Cambridge, UK) and the expression of Acyl-Coenzyme A oxydase 1 (ACOX1), a target gene of PPAR $\alpha$, was determined in hepatic samples obtained as previously described [23], using a mouse antibody against ACOX1 (Santa Cruz Biotechnology, Santa Cruz, CA, USA). Both antibodies were incubated overnight at $4{ }^{\circ} \mathrm{C}$ followed by incubation with rabbit anti-mouse HRP-conjugated secondary antibody (1:10,000, $1 \mathrm{~h}$, room temperature; Stressgen, Victoria, BC, Canada). Blots were revealed by chemiluminiscence. Protein expression was determined by densitometric analysis using the Science Lab, Image Gauge (Fuji Photo Film GMBH, Düsseldorf). Quantitative densitometric values were normalized to TATA-binding protein (TBP; Abcam) for PPAR $\alpha$ or to GAPDH (Sta Cruz) for ACOX.

Electrophoretic mobility shift assays (EMSA)

Electrophoretic mobility shift assay (EMSA) to evaluate PPAR $\alpha$ binding activity was performed as described previously [24]. The DNA sequence of the doublestranded oligonucleotide used was: consensus binding site of PPAR response element, 5'-CAAAACTAGGTCAAAGGTCA-3' (Santa Cruz Biotec.). Oligonucleotides were labeled and then, to separate the labeled probe from the unbound ATP the reaction mixture was eluted in a Nick column (Amersham Biosciences) following the manufacturer's instructions. Eight micrograms of crude nuclear proteins was incubated for $10 \mathrm{~min}$ on ice in binding buffer $(10 \mathrm{mM}$ Tris- $\mathrm{HCl} \mathrm{pH}$ 8.0, $25 \mathrm{mM} \mathrm{KCl}, 0.5 \mathrm{mM}$ DTT, $0.1 \mathrm{mM}$ EDTA pH 8.0, 5\% glycerol, $5 \mathrm{mg} / \mathrm{ml}$ BSA, and $50 \mu \mathrm{g} / \mathrm{ml}$ poly(dI-dC)), in a final volume of $15 \mu \mathrm{l}$. The labeled probe (approximately 55,000 cpm) was added and the reaction was incubated for $20 \mathrm{~min}$ at room temperature. Moreover, for supershift assays, antibodies were added before incubation with the labeled probe for a further $30 \mathrm{~min}$ at $4{ }^{\circ} \mathrm{C}$. The involvement of PPAR $\alpha$ in the formation of specific bands is shown by the appearance of three marked supershifted bands when hepatic nuclear extracts were co-incubated with PPAR $\alpha$ antibody, whereas Oct- 1 antibody was used to demonstrate that supershifts were not due to an unspecific interference produced by the presence in the incubation medium of immunoglobulin proteins. Protein-DNA complexes were resolved by electrophoresis at $4{ }^{\circ} \mathrm{C}$ on a $5 \%$ acrylamide gel and subjected to autoradiography. PPAR $\alpha$ and octamer motif- 1 transcription factor (Oct-1) antibodies were from Santa Cruz Biotec.

In vivo hemodynamic studies

Rats were anesthetised with ketamine hydrochloride $(100 \mathrm{mg} / \mathrm{kg}$; Merial Laboratories, Barcelona, Spain) plus midazolam ( $5 \mathrm{mg} / \mathrm{kg}$; Laboratorios Reig Jofré, Barcelona, Spain) intraperitoneally. PE-50 catheters were introduced into the femoral artery to measure mean arterial pressure (MAP; $\mathrm{mmHg}$ ) and into the ileocolic vein to measure portal pressure (PP, $\mathrm{mmHg}$ ). Perivascular ultrasonic flow probes connected to a flowmeter (Transonic Systems Inc., Ithaca, NY, USA) were placed around the portal vein, as close as possible to the liver to avoid portal-collateral blood flow, to measure portal blood flow (PBF; $\mathrm{ml} \cdot \mathrm{min}^{-1}$ ) perfusing the liver. Blood pressures and flows were registered on a multichannel computer-based recorder (PowerLab; AD Instruments, Colorado Springs, CO). The temperature of the animals was maintained at $37 \pm 0.5^{\circ} \mathrm{C}$ and hemodynamic data were collected after a 20 -min stabilization period.

\section{Biochemical measurements}

At the end of data collection, blood samples from a peripheral vein were taken for biochemical analysis. Serum was separated within $15 \mathrm{~min}$ and frozen at $-70{ }^{\circ} \mathrm{C}$ for subsequent analysis. Cholesterol, AST, ALT, albumin, and triglycerides (TG) levels were determined by current standard protocols.

Evaluation of endothelial function

After in vivo hemodynamic measurements, livers were quickly isolated and perfused by a flow-controlled perfusion system as previously described [25]. The perfused rat liver preparation was allowed to stabilize for 20 min before vasoactive substances were added. The intrahepatic microcirculation was preconstricted by adding the $\alpha_{1}$-adrenergic agonist methoxamine (Mtx; $10^{-4} \mathrm{~mol} / \mathrm{L} ; \mathrm{Sigma}$ ) to the reservoir. After $5 \mathrm{~min}$, concentration-response curves to cumulative doses of acetylcholine (Ach; $10^{-7}, 10^{-6}$, and $10^{-5} \mathrm{~mol} / \mathrm{L}$; Sigma) were evaluated. Responses to Ach were calculated as per cent change in portal perfusion pressure [3]. The gross appearance of the liver, stable perfusion pressure, bile production over $0.4 \mu \mathrm{l} / \mathrm{min} / \mathrm{g}$ of liver and a stable buffer $\mathrm{pH}(7.4 \pm 0.1)$ were monitored during this period. If any viability criteria were not satisfied, the experiment was discarded.

Evaluation of NO bioavailability

Isolation and culture of hepatic endothelial cells (HEC)

HEC were isolated from control and cirrhotic rats as previously described [26,27]. Briefly, after collagenase perfusion of the livers and isopycnic sedimentation of the resulting dispersed cells through a two-step density gradient of Percoll, pure monolayer cultures of HEC were established by selective attachment on a substrate of rat tail collagen type I. Afterwards, cells were cultured in Roswell Park Memorial Institute (RPMI)-1640 medium and studies were performed on cells from the first passage, $12 \mathrm{~h}$ after their isolation, to preserve their typical phenotype [28].

$P P A R \alpha$ activation in HEC

To determine whether PPAR $\alpha$ activation could increase NO bioavailability in HEC isolated from cirrhotic rat livers (CH-HEC), $\mathrm{CH}-\mathrm{HEC}$ were incubated for $30 \mathrm{~min}$ at $37^{\circ} \mathrm{C}$ with vehicle (DMSO) or with fenofibrate $(100 \mu \mathrm{M})$. Then, we evaluated NO levels by DAF-FM-DA staining and eNOS activation by Western blot in different subsets of $\mathrm{CH}-\mathrm{HEC}$

Measurement of NO levels in HEC

In situ NO levels in CH-HEC were assessed using 4-amino-5-methylamino-2', $7^{\prime}-$ difluorofluorescein diacetate (DAF-FM-DA) (Molecular Probes, Inc., Eugene, OR) on a laser scanning confocal microscope (model TCS-SL DMIRE2, Leica, Wetzlar, Germany) as described [26]. Quantitative analysis was obtained by averaging the peak relative fluorescent intensity (optical density arbitrary units) of each cell using Image J $1.43 \mathrm{~m}$ software (NIH, USA) and normalization of the fluorescent result by the total number of cultured cells counted from each corresponding digitalized phase contrast microscope image. 
Table 1. Effects of fenofibrate treatment on metabolic parameters.

\begin{tabular}{llll}
\hline Parameter & $\begin{array}{l}\mathrm{CH} \text { vehicle } \\
\mathrm{n}=10\end{array}$ & $\begin{array}{l}\mathrm{CH} \text { fenofibrate } \\
\mathrm{n}=10\end{array}$ & $p$ \\
\hline Body weight $(\mathrm{g})$ & $341 \pm 41$ & $379 \pm 83$ & 0.12 \\
Serum cholesterol $(\mathrm{mg} / \mathrm{dl})$ & $89 \pm 7$ & $65 \pm 7$ & 0.03 \\
Serum triglycerides $(\mathrm{mg} / \mathrm{dl})$ & $43 \pm 16$ & $34 \pm 17$ & 0.2 \\
ALT (IU/L) & $99 \pm 7$ & $101 \pm 17$ & 0.92 \\
AST (IU/L) & $240 \pm 28$ & $225 \pm 43$ & 0.77 \\
Albumin (g/L) & $29 \pm 3$ & $28 \pm 3$ & 0.81 \\
\hline
\end{tabular}

Data are means $\pm \mathrm{SD}$. $\mathrm{CH}$ vehicle denotes cirrhotic rats receiving vehicle, and $\mathrm{CH}$ fenofibrate denotes cirrhotic rats receiving fenofibrate.

eNOS activation in HEC

eNOS activation was determined by eNOS phosphorylation/eNOS total ratio by Western blot in CH-HEC treated with fenofibrate $(n=6)$ or vehicle $(n=6)$ as previously described [29].

\section{cGMP levels}

Measurements of cGMP, a marker of NO bioavailability, were performed in rat liver homogenates from cirrhotic rats treated with fenofibrate $(n=8)$ or vehicle $(n=8)$ by enzyme immunoassay as previously described $[19,25]$.

Evaluation of COX pathway

Protein expression of $\mathrm{COX}-1$

COX-1 expression was determined in hepatic samples using a mouse antibody against COX-1 (Cayman Chem Co., Tallin, Estonia).

Measurement of thromboxane $B_{2}$

In liver-perfusion experiments, samples of the perfusate were obtained before Mtx administration and after the dose-response to Ach. The samples were stored at $-80^{\circ} \mathrm{C}$ and thromboxane $\mathrm{B}_{2}\left(\mathrm{TXB}_{2}\right)$, the end metabolite of $\mathrm{TXA}_{2}$, was quantified in duplicate using a commercially available enzyme immunoassay (Cayman Chem Co.) [30]. $\mathrm{TXB}_{2}$ production was expressed as absolute increment over baseline before Mtx administration and after dose-response curve to Ach.

Evaluation of hepatic fibrosis

Quantification of hepatic fibrosis

Livers from cirrhotic rats were fixed in $10 \%$ formalin, embedded in paraffin, sectioned and stained with $0.1 \%$ Sirius Red, photographed, and analyzed using a microscope equipped with a digital camera. Six fields from each slide were randomly selected, and the red-stained area per total area was measured using AxioVision software [31]. Values are expressed as the mean of 30-42 fields taken from 5 to 7 animals per group.

Collagen I and MMPS/TIMPs gene expression

Hepatic mRNA expression of collagen I, MMP2, MMP9, MMP13, TIMP1, and TIMP2 was analyzed using predesigned gene expression assays obtained from Applied Biosystems as described above.

Protein expression of $\alpha S M A$

Hepatic protein expression of $\alpha$-smooth muscle actin ( $\alpha \mathrm{SMA}$ ) was determined by Western blot in hepatic samples using a mouse antibody against $\alpha$ SMA (Sigma).

Statistical analysis

Statistical analysis was performed using SPSS 16.0 for Windows statistical package (SPSS Inc., Chicago, IL). All results are expressed as mean \pm SD in the text whereas in figures are represented by boxplots with median and interquartile range. Comparisons between groups were performed with the Student's $t$-test or Mann-Whitney $t$-test for unpaired data when adequate. Differences were considered significant at a $p$ value $<0.05$.

\section{JOURNAL OF HEPATOLOGY}

\section{Results}

All rats had macroscopic cirrhosis and signs of portal hypertension. Weight gain during the study was similar between fenofibrate-treated and vehicle-treated rats. No rat died during treatment. There were no significant differences in AST, ALT, and albumin levels between the two groups of cirrhotic rats (Table 1). Rats treated with fenofibrate had significanly lower serum cholesterol levels. Serum triglyceride levels were also lower, although they did not reach statistical significance probably because of the small sample size (Table 1 ).

\section{Fenofibrate treatment activates PPAR $\alpha$ in $\mathrm{CCl}_{4}$-cirrhotic rats}

$\mathrm{CCl}_{4}$-cirrhotic rats treated with fenofibrate had an increase in $P P A R \alpha$ mRNA expression (Fig. 1A) and in PPAR $\alpha$ protein expression (Fig 1A). Fig. 1B shows the pattern of retardation bands obtained in EMSA, performed by incubating a PPRE oligonucleotide probe and hepatic nuclear extracts obtained from vehicleand fenofibrate-treated cirrhotic rats. An enhanced PPAR $\alpha$ binding activity was demonstrated when comparing the intensity of the shifted bands (IC) between samples from vehicle- and fenofibrate-treated cirrhotic rats. Fenofibrate treatment produced a marked significant increase in the intensity of bands II and III (Fig. 1C). Increased PPAR $\alpha$ activity was associated with an augmented protein expression of one of its target genes, ACOX1 (Fig. 1D).

\section{PPAR $\alpha$ activation lowers portal pressure in $\mathrm{CCl}_{4}$-cirrhotic rats}

Portal pressure (PP) was significantly lower in cirrhotic rats treated with fenofibrate than in those treated with vehicle (mean decrease 29\%) (Table 2), without significant changes in PBF. In addition, MAP was significantly higher in cirrhotic rats treated with fenofibrate than in rats treated with vehicle (Table 2 ).

$P P A R \propto$ activation improves endothelial dysfunction in $\mathrm{CCl}_{4}$-cirrhotic rats

Baseline portal perfusion pressure was significantly lower in livers from cirrhotic rats treated with fenofibrate than in those treated with vehicle $(7.4 \pm 1.2 \mathrm{~mm} \mathrm{Hg} v s .10 .3 \pm 1.9 \mathrm{~mm} \mathrm{Hg} ; p<0.05)$

As expected, livers from cirrhotic rats treated with vehicle exhibited an impaired vasodilatory response to Ach. Fenofibrate treatment significantly improved vasorelaxation in response to Ach (Fig. 2A).

\section{PPAR $\alpha$ activation increases NO bioavailability in HEC}

We assessed NO bioavailability by DAF-FM-DA staining in HEC isolated from cirrhotic rat livers (CH-HEC). Fenofibrate administration promoted a significant increase in NO in CH-HEC (Fig. 2B). This was associated with a trend to increase eNOS phospho/total ratio in CH-HEC $(1 \pm 0.49$ vs. $2.49 \pm 2.34 ; p=0.1)$ and cGMP levels in the whole liver (19.6 \pm 6.7 vs. $24.3 \pm 5.1 ; p=0.16$ ).

PPAR $\alpha$ activation attenuates $\mathrm{COX}-1$ activation in $\mathrm{CCl}_{4}$-cirrhotic rats

COX-1 protein expression was significantly reduced in cirrhotic livers treated with fenofibrate as compared with those treated with vehicle (Fig. $3 \mathrm{~A}$ ). In addition, $\mathrm{TXB}_{2}$ was significantly dimin- 
Research Article

A

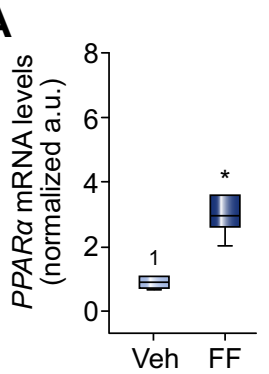

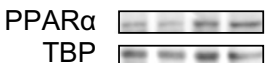

TBP $-=-$

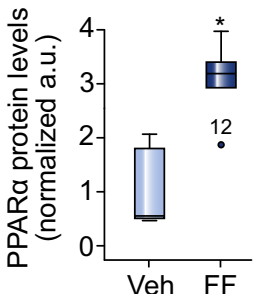

B

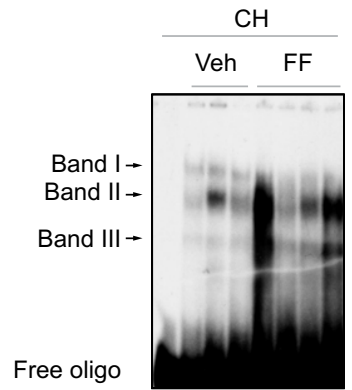

C

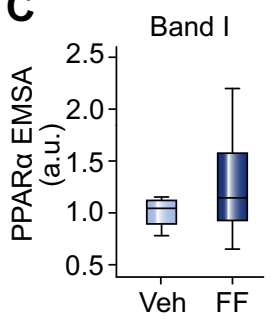

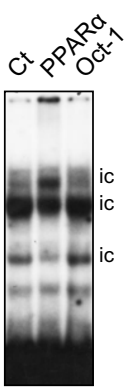

Band III
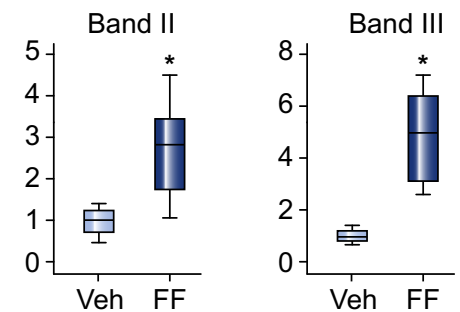

D

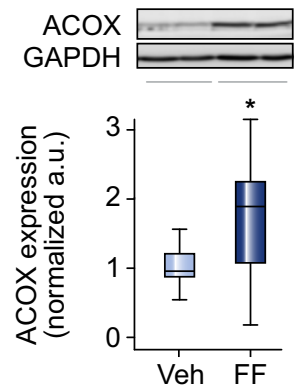

Fig. 1. Effects of fenofibrate treatment on PPAR $\alpha$ activation. (A) Left panel: relative PPAR $\alpha$ mRNA levels in hepatic samples from $\mathrm{CCl}_{4}$-cirrhotic rats treated with vehicle $(n=11 ;$ Veh) or fenofibrate $(n=10 ; F F)$ normalized to an endogenous reference gene (18S). Values (median \pm interquartile range) are normalized to Veh-treated liver expression. Right panel: representative Western blot image of PPAR $\alpha$ in nuclear extracts from $\mathrm{CCl}_{4}$-cirrhotic rat livers treated with vehicle (Veh; $n=6)$ or fenofibrate $(F F ; n=6)$. Densitometric analysis in arbitrary units, normalized to TATA-binding protein (TBP), showed an increased expression of PPAR $\alpha$ in fenofibrate-treated $\mathrm{CCl}_{4}$-cirrhotic rat livers. (B) The left side of the figure consists of a representative EMSA autoradiography, showing the pattern of retardation bands obtained by incubating hepatic nuclear extracts from $\mathrm{CCl}_{4}$-cirrhotic rats treated with vehicle $(\mathrm{n}=4$; Veh) or fenofibrate $(n=6 ; F F)$ with a PPRE oligonucleotide, forming at least three specific bands (bands I-III). In the right part of the figure, the involvement of PPAR $\alpha$ in the formation of specific bands is shown by the appearance of marked supershifted (IC) bands when hepatic nuclear extracts were coincubated with PPAR $\alpha$ antibody (Ab). Oct- $1 \mathrm{Ab}$ was used to demonstrate that supershifts were not due to an unspecific interference produced by the presence in the incubation medium of immunoglobulin proteins. (C) Box plot
Table 2. Effects of fenofibrate treatment on systemic and hepatic hemodynamics.

\begin{tabular}{llll}
\hline Parameter & $\begin{array}{l}\mathrm{CH} \text { vehicle } \\
\mathrm{n}=10\end{array}$ & $\begin{array}{l}\mathrm{CH} \text { fenofibrate } \\
\mathrm{n}=8\end{array}$ & $p$ \\
\hline $\mathrm{PP}(\mathrm{mmHg})$ & $17.1 \pm 2.5$ & $12.2 \pm 1.7$ & 0.001 \\
PBF $(\mathrm{ml} / \mathrm{min})$ & $14.6 \pm 1.8$ & $11.6 \pm 1.4$ & 0.21 \\
MAP $(\mathrm{mmHg})$ & $83 \pm 15$ & $103 \pm 20$ & 0.02 \\
HR $(\mathrm{bpm})$ & $320 \pm 36$ & $354 \pm 48$ & 0.48 \\
\hline
\end{tabular}

Data are means \pm SD. MAP, mean arterial pressure; PP, portal pressure; PBF, portal blood flow; HR, heart rate. $\mathrm{CH}$ vehicle denotes cirrhotic rats receiving vehicle, and $\mathrm{CH}$ fenofibrate denotes cirrhotic rats receiving fenofibrate.

A

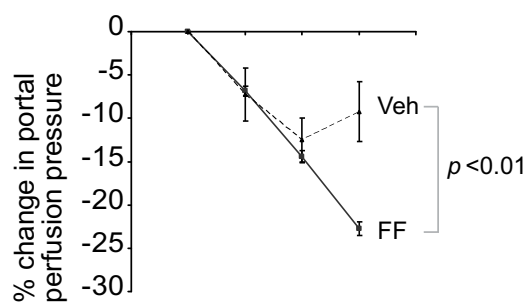

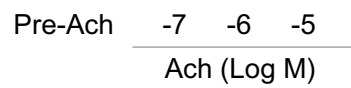

B

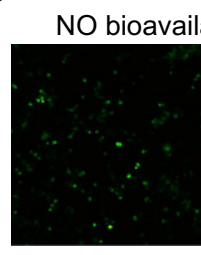

Veh

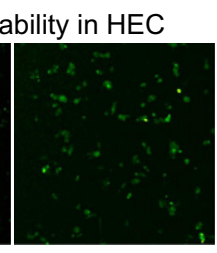

FF

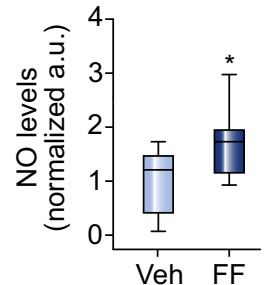

Fig. 2. Effect of PPARa activation on endothelial function of $\mathbf{C C l} 4$ cirrhotic rats. (A) Endothelium-dependent vasorelaxation to acetylcholine (Ach) in isolated and perfused livers of $\mathrm{CCl}_{4}$-cirrhotic rats treated with vehicle (Veh; $\mathrm{n}=7$ ) or fenofibrate (FF; $n=7$ ). Results are expressed as mean \pm SEM. Fenofibrate treatment significantly improved the impaired vasodilatory response to Ach in $\mathrm{CCl}_{4}$ cirrhotic rat livers. PP, portal perfusion pressure. (B) Effects of PPAR $\alpha$ activation on NO bioavailability in hepatic endothelial cells. Left panel, fluorescent detection of intracellular nitric oxide (NO) in hepatic endothelial cells (HEC) isolated from cirrhotic rat livers (CH-HEC). Representative images (confocal microscopy) of DAF-FM-DA fluorescence from CH-SEC treated with vehicle (Veh) or fenofibrate (FF). Original magnification, $40 \times$; bar $10 \mu \mathrm{m}$. Right panel, the fluorescence intensity of DAF-FM-DA in arbitrary units was normalized to the total number of cells. The data shown are from 4873 individual Veh- and 4196 fenofibrate-CH-SEC obtained from three different experiments. Fenofibrate administration promoted a significant increase in NO bioavailability; ${ }^{*} p<0.01$ vs. Veh-treated CH-SEC.

ished in the perfusate of cirrhotic livers treated with fenofibrate in comparison to those treated with vehicle (Fig. 3B).

PPAR $\alpha$ activation attenuates intrahepatic fibrosis in $\mathrm{CCl}_{4}$-cirrhotic rats

As expected, cirrhotic rats exhibited a marked architectural distortion with abundant fibrosis revealed by extensive collagen

analysis of the relative intensity of the specific PPAR $\alpha$ bands I, II, and III. (D) Representative Western blot image of ACOX1 in $\mathrm{CCl}_{4}$-cirrhotic rat livers treated with vehicle (Veh; $n=6$ ) or fenofibrate (FF; $n=8$ ) and densitometric analysis in arbitrary units, normalized to glyceraldehyde 3-phosphate dehydrogenase (GAPDH), showed an increased expression of ACOX in fenofibrate-treated $\mathrm{CCl}_{4}$-cirrhotic rat livers. 
A
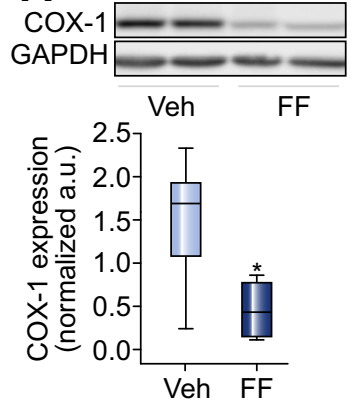

B

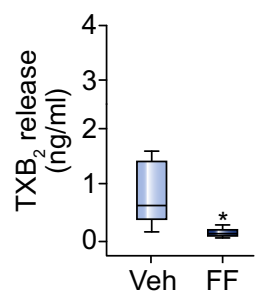

Fig. 3. Effects of PPAR $\alpha$ activation on COX-1 activation in $\mathbf{C C l}_{4}$-cirrhotic rats (A) Representative Western blot image of $\mathrm{COX}-1$ in $\mathrm{CCl}_{4}$-cirrhotic rat livers treated with vehicle (Veh; $n=6)$ or fenofibrate (FF; $n=8$ ) and densitometric analysis in arbitrary units, normalized to glyceraldehyde 3-phosphate dehydrogenase (GAPDH), showed a decreased expression of COX-1 in fenofibrate-treated $\mathrm{CCl}_{4}$-cirrhotic rat livers. (B) $\mathrm{TXB}_{2}$ production in Veh- or fenofibrate-treated $\mathrm{CCl}_{4}$ cirrhotic rat livers. Values represent median \pm interquartile range; ${ }^{*} p<0.01 v s$. vehicle-treated $\mathrm{CCl}_{4}$-cirrhotic rats.

staining. Importantly, rats receiving fenofibrate had a 50\% reduction in collagen deposition resulting in thinner septa and larger regenerating nodules than vehicle-treated cirrhotic animals (Fig. 4A). This was associated with a significant decrease in type I collagen mRNA levels (Fig. 4B) and with a marked decrease in aSMA expression, a surrogate marker of hepatic stellate cell activation (Fig. 4C). The resolution of fibrosis is also supported by

\section{JOURNAL OF HEPATOLOGY}

decreased MMP2, MMP9, MMP13, TIMP1, and TIMP2 mRNA levels (Fig. 4D).

\section{Discussion}

Increased resistance to portal blood flow is the primary factor leading to portal hypertension in cirrhosis [1]. Much of this increase is determined by structural changes in the liver and is further aggravated by an increase in hepatic vascular tone. In a healthy liver, the endothelium is able to release vasoactive substances to properly modulate intrahepatic hemodynamics. By contrast, the hepatic vascular bed of cirrhotic livers exhibits endothelial dysfunction, which represents the first sign of microvascular injury at the organ level [32].

Substantial data suggest that PPAR $\alpha$ regulates processes that are involved in the regulation of vascular function [33]. Importantly, PPAR $\alpha$ is expressed in the liver and in vascular endothelial cells [34], suggesting that PPAR $\alpha$ activation may exert beneficial effects on the vascular wall [35] also in the liver. Fibrates activate $\operatorname{PPAR} \alpha$, which results in a wide spectrum of downstream effects, including regulation of target genes related to the pathophysiological mechanisms involved in the development of portal hypertension in cirrhosis, such as vascular tone, oxidative stress, and fibrosis.

Our study clearly shows that fenofibrate treatment reduces fibrosis, improves hepatic endothelial dysfunction and reduces PP in cirrhotic rats. Importantly, PP was 29\% lower in cirrhotic
A

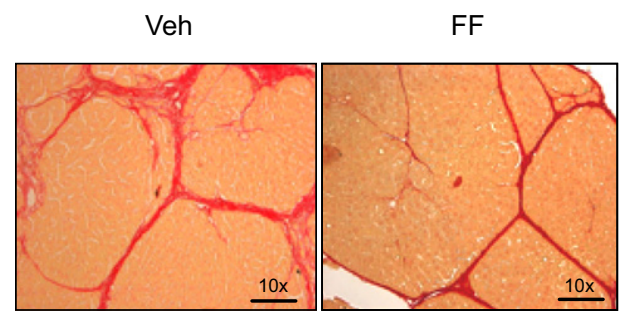

D

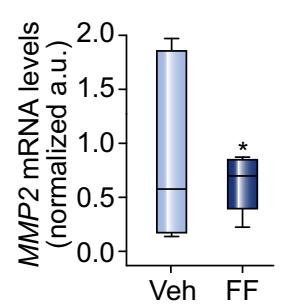

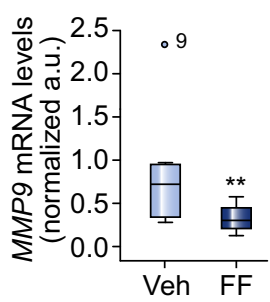

B
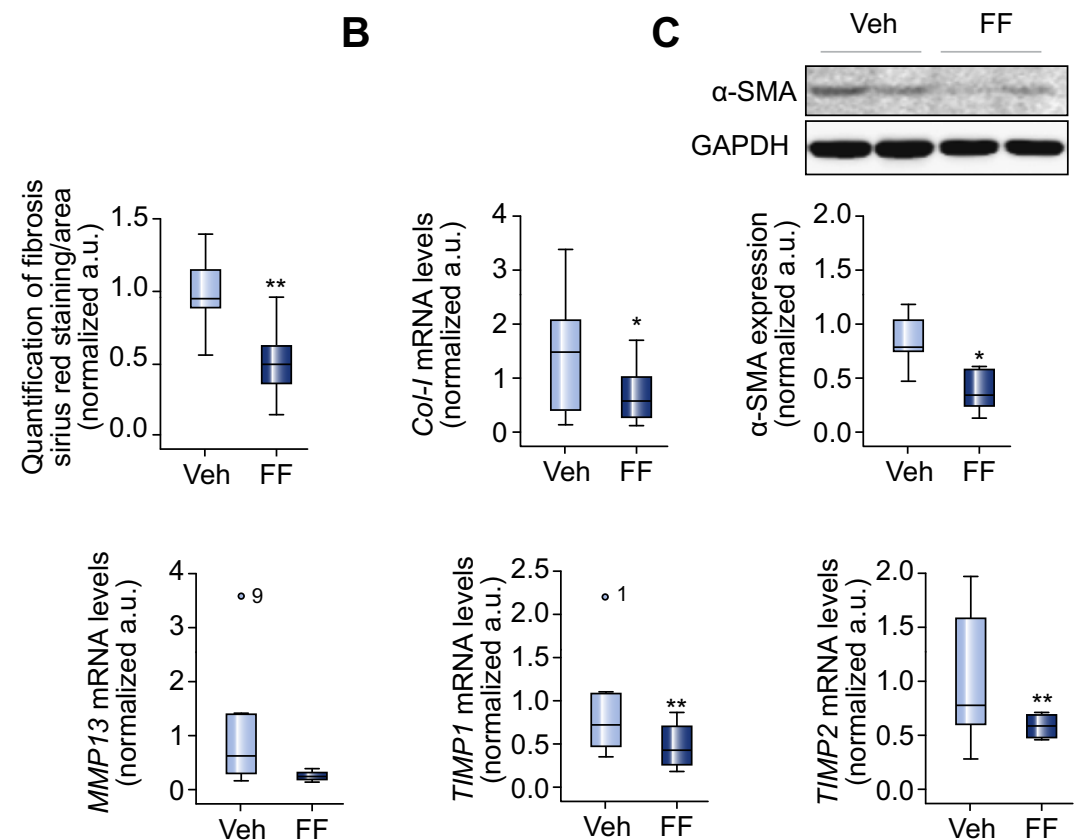

Fig. 4. Effects of PPAR $\boldsymbol{\alpha}$ activation on hepatic fibrosis in $\mathbf{C C l}_{\mathbf{4}}$-cirrhotic rats. (A) Left panel, representative histological images of livers stained with Sirius Red from fenofibrate (FF)- or vehicle (Veh)- treated $\mathrm{CCl}_{4}$-cirrhotic rats (original magnification $50 \times$ ). Right panel, quantification of liver fibrosis (Sirius Red staining area per total area) in fenofibrate- or Veh-treated $\mathrm{CCl}_{4}$-cirrhotic rats, ${ }^{* *} p<0.01$. (B) Collagen I mRNA expression levels in $\mathrm{CCl}_{4}$-cirrhotic rat livers treated with Veh $(\mathrm{n}=11)$ or fenofibrate $(\mathrm{n}=10$ ) normalized to an endogenous reference gene $(G A P D H)$. Values (median \pm interquartile range) are normalized to Veh-treated livers expression. (C) Representative Western blot analysis of $\alpha$ SMA in livers from Veh $(n=6)$ or fenofibrate-treated $(n=8) C H$ rats and densitometric quantification in arbitrary units (AU), normalized to GAPDH, showed a decrease of $\alpha$ SMA expression in fenofibrate-treated $\mathrm{CCl}_{4}$-cirrhotic rats; ${ }^{*} p<0.05$ vs. vehicle-treated $\mathrm{CCl}_{4}$-cirrhotic rats. (D) MMP2, MMP9, MMP13, TIMP1, and TIMP2 mRNA expression levels in $\mathrm{CCl}_{4}$-cirrhotic rat livers treated with Veh $(\mathrm{n}=11)$ or fenofibrate $(\mathrm{n}=10)$ normalized to an endogenous reference gene $(\mathrm{GAPDH})$. Values (median \pm interquartile range) are normalized to Veh-treated livers expression; ${ }^{*} p<0.05,{ }^{* *} p<0.01$. 


\section{Research Article}

rats receiving fenofibrate than in those receiving vehicle. It is worth remembering that this reduction is well above the $20 \%$ target reduction that has been associated with an improvement in prognosis in patients with cirrhosis [36].

Our results suggest that reduction in PP is mainly due to the effect of fenofibrate improving the architectural abnormalities of the cirrhotic liver. Histopathological analysis revealed that fenofibrate markedly atttenuates intrahepatic fibrosis. Specifically, we observed a robust attenuation of septal thickness and an increase in nodule size. It is important to emphasize that wider septal thickness and lower nodule size have been shown to be associated with a more severe degree of portal hypertension [37]. Further reinforcing the concept that fenofibrate reduced fibrosis was the observed significant reduction in collagen I mRNA expression and $\alpha$-SMA protein expression (a surrogate of HSC activation). These findings are in agreement with previous studies showing that PPAR $\alpha$ activation is implicated in the regulation of fibrosis in other experimental models [38]. In fact, PPAR $\alpha$ activation prevents fibrosis in a TAA-induced hepatic fibrosis model [18].

Fenofibrate also improves intrahepatic endothelial dysfunction. We have previously shown that endothelial dysfunction in cirrhotic livers is due, at least in part, to an increased production of COX-1-derived prostanoids [3]. Interestingly, a previous study reported that fenofibrate has a protective effect in an experimental model of age-associated endothelial dysfunction, by reducing the expression of COX-1 and the release of $\mathrm{TXA}_{2}$ [17]. Our study suggests that this also happens in $\mathrm{CCl}_{4}$-cirrhotic rats, where fenofibrate treatment was associated with a reduction of COX-1 protein expression and $\mathrm{TXA}_{2}$ production. To what extent this effect on the COX signalling pathway represents a direct effect of fenofibrate or it is due to improvement of the hepatic architecture remains conjectural but it is likely that both mechanisms are involved.

A second factor involved in hepatic endothelial dysfunction in cirrhosis is a reduced intrahepatic NO availability. Fenofibrate incubation promoted a significant increase in NO bioavailability in endothelial cells isolated from cirrhotic livers that was associated with a trend for an increase in eNOS activation in endothelial cells and cGMP levels in the whole tissue.

Another important finding of the present study is the effect of fenofibrate increasing arterial pressure in cirrhotic rats. It is reasonable to hypothesize that improved hepatic hemodynamics in cirrhosis may have beneficial effects on the splanchnic and systemic circulation, by deactivating factors triggered by the increased intrahepatic resistance. In that regard, we and others have previously observed a similar improvement in systemic hypotension in studies where NO availability has been improved by tetrahydrobiopterin supplementation [39] or by transducing actived-Akt into the liver of $\mathrm{CCl}_{4}$-cirrhotic rats [40]. In any case, the increase in arterial pressure represents an additional benefit of fenofibrate treatment.

Fenofibrate has been extensively used in the treatment of dyslipidemia, since it markedly decreases cholesterol levels and has an excellent safety profile [41]. In addition, pilot studies in patients with primary biliary cirrhosis and non-alcoholic fatty liver disease suggest that this drug is safe in patients with liver diseases, in whom it may improve liver function [42,43].

In conclusion, our data provides evidence that PPAR $\alpha$ activation may have beneficial effects in several mechanisms involved in the pathophysiology of portal hypertension, leading to a regression of liver fibrosis in cirrhotic rats, to a correction of endothelial dysfunction, to a reduction of portal pressure and to an amelioration of systemic hypotension. This new therapeutic strategy for portal hypertension should obviously be confirmed in patients with cirrhosis.

\section{Conflict of interest}

The authors who have taken part in this study declared that they do not have anything to disclose regarding funding or conflict of interest with respect to this manuscript.

\section{Financial Support}

This study was supported by Grants from the Ministerio de Educación y Ciencia (SAF2010/17043) and from Instituto de Salud Carlos III (PS 09/01261). Ciberehd is funded by Instituto de Salud Carlos III.

\section{Acknowledgements}

Part of this work was carried out at the Esther Koplowitz Centre, Barcelona. The authors are in debt to Montse Monclús for technical assistance.

\section{References}

[1] Bosch J, Garcia-Pagan JC. Complications of cirrhosis. I. Portal hypertension. J Hepatol 2000;32:141-156.

[2] Graupera M, Garcia-Pagan JC, Abraldes JG, Peralta C, Bragulat M, Corominola $\mathrm{H}$, et al. Cyclooxygenase-derived products modulate the increased intrahepatic resistance of cirrhotic rat livers. Hepatology 2003;37:172-181.

[3] Graupera M, Garcia-Pagan JC, Pares M, Abraldes JG, Rosello J, Bosch J, et al. Cyclooxygenase- 1 inhibition corrects endothelial dysfunction in cirrhotic rat livers. J Hepatol 2003;39:515-521.

[4] Wiest R, Groszmann RJ. The paradox of nitric oxide in cirrhosis and portal hypertension: too much, not enough. Hepatology 2002;35:478-491.

[5] Auboeuf D, Rieusset J, Fajas L, Vallier P, Frering V, Riou JP, et al. Tissue distribution and quantification of the expression of mRNAs of peroxisome proliferator-activated receptors and liver $\mathrm{X}$ receptor-alpha in humans: no alteration in adipose tissue of obese and NIDDM patients. Diabetes 1997;46:1319-1327.

[6] Mandard S, Muller M, Kersten S. Peroxisome proliferator-activated receptor alpha target genes. Cell Mol Life Sci 2004;61:393-416.

[7] Kersten S, Desvergne B, Wahli W. Roles of PPARs in health and disease. Nature 2000;405:421-424.

[8] Robinson E, Grieve DJ. Significance of peroxisome proliferator-activated receptors in the cardiovascular system in health and disease. Pharmacol Ther 2009;122:246-263.

[9] Berger J, Moller DE. The mechanisms of action of PPARs. Annu Rev Med 2002;53:409-435.

[10] Iglarz M, Touyz RM, Viel EC, Paradis P, Amiri F, Diep QN, et al. Peroxisome proliferator-activated receptor-alpha and receptor-gamma activators prevent cardiac fibrosis in mineralocorticoid-dependent hypertension. Hypertension 2003;42:737-743.

[11] Kanie N, Matsumoto T, Kobayashi T, Kamata K. Relationship between peroxisome proliferator-activated receptors (PPAR alpha and PPAR gamma) and endothelium-dependent relaxation in streptozotocin-induced diabetic rats. Br J Pharmacol 2003;140:23-32.

[12] Tabernero A, Schoonjans K, Jesel L, Carpusca I, Auwerx J, Andriantsitohaina R. Activation of the peroxisome proliferator-activated receptor alpha protects against myocardial ischaemic injury and improves endothelial vasodilatation. BMC Pharmacol 2002;2:10.

[13] Hamblin M, Chang L, Fan Y, Zhang J, Chen YE. PPARs and the cardiovascular system. Antioxid Redox Signal 2009;11:1415-1452. 
[14] Murakami H, Murakami R, Kambe F, Cao X, Takahashi R, Asai T, et al. Fenofibrate activates AMPK and increases eNOS phosphorylation in HUVEC. Biochem Biophys Res Commun 2006;341:973-978.

[15] Okayasu T, Tomizawa A, Suzuki K, Manaka K, Hattori Y. PPARalpha activators upregulate eNOS activity and inhibit cytokine-induced NF-kappaB activation through AMP-activated protein kinase activation. Life Sci 2008;82:884-891.

[16] Watts GF, Staels B. Regulation of endothelial nitric oxide synthase by PPAR agonists: molecular and clinical perspectives. Arterioscler Thromb Vasc Biol 2004;24:619-621.

[17] Álvarez de Sotomayor M, Mingorance C, Andriantsitohaina R. Fenofibrate improves age-related endothelial dysfunction in rat resistance arteries. Atherosclerosis 2007;193:112-120.

[18] Toyama T, Nakamura H, Harano Y, Yamauchi N, Morita A, Kirishima T, et al. PPARalpha ligands activate antioxidant enzymes and suppress hepatic fibrosis in rats. Biochem Biophys Res Commun 2004;324:697-704.

[19] Garcia-Caldero H, Rodriguez-Vilarrupla A, Gracia-Sancho J, Divi M, Lavina B, Bosch J, et al. Tempol administration, a superoxide dismutase mimetic, reduces hepatic vascular resistance and portal pressure in cirrhotic rats. J Hepatol 2010;54:660-665.

[20] Ichihara S, Obata K, Yamada Y, Nagata K, Noda A, Ichihara G, et al. Attenuation of cardiac dysfunction by a PPAR-alpha agonist is associated with down-regulation of redox-regulated transcription factors. J Mol Cell Cardiol 2006;41:318-329.

[21] Alegret M, Ferrando R, Vazquez M, Adzet T, Merlos M, Laguna JC. Relationship between plasma lipids and palmitoyl-CoA hydrolase and synthetase activities with peroxisomal proliferation in rats treated with fibrates. Br J Pharmacol 1994;112:551-556.

[22] Sanguino E, Roglans N, Alegret M, Sanchez RM, Vazquez-Carrera M, Laguna JC. Different response of senescent female Sprague-Dawley rats to gemfibrozil and rosiglitazone administration. Exp Gerontol 2005;40:588-598.

[23] Abraldes JG, Graupera M, Zafra C, Rodriguez-Vilarruplaa A, Fernandez M, Garcia-Pagan JC, et al. Simvastatin improves sinusoidal endothelial dysfunction in CCl4 cirrhotic rats. J Hepatol 2005;42:62-63.

[24] Sanguino E, Ramon M, Michalik L, Wahli W, Alegret M, Sanchez RM, et al Lack of hypotriglyceridemic effect of gemfibrozil as a consequence of agerelated changes in rat liver PPARalpha. Biochem Pharmacol 2004;67: 157-166.

[25] Lavina B, Gracia-Sancho J, Rodriguez-Vilarrupla A, Chu Y, Heistad DD, Bosch $\mathrm{J}$, et al. Superoxide dismutase gene transfer reduces portal pressure in $\mathrm{CCl} 4$ cirrhotic rats with portal hypertension. Gut 2009;58:118-125.

[26] Gracia-Sancho J, Lavina B, Rodriguez-Vilarrupla A, Garcia-Caldero H, Fernandez $\mathrm{M}$, Bosch J, et al. Increased oxidative stress in cirrhotic rat livers: a potential mechanism contributing to reduced nitric oxide bioavailability. Hepatology 2008;47:1248-1256.

[27] Braet F, De Zanger R, Sasaoki T, Baekeland M, Janssens P, Smedsrod B, et al. Assessment of a method of isolation, purification, and cultivation of rat liver sinusoidal endothelial cells. Lab Invest 1994;70:944-952.

[28] DeLeve LD, Wang X, Hu L, McCuskey MK, McCuskey RS. Rat liver sinusoidal endothelial cell phenotype is maintained by paracrine and autocrine regulation. Am J Physiol Gastrointest Liver Physiol 2004;287:G757-G763.

\section{JOURNAL OF HEPATOLOGY}

[29] Abraldes JG, Rodriguez-Vilarrupla A, Graupera M, Zafra C, Garcia-Caldero H, Garcia-Pagan JC, et al. Simvastatin treatment improves liver sinusoidal endothelial dysfunction in $\mathrm{CCl}(4)$ cirrhotic rats. J Hepatol 2007;46: 1040-1046.

[30] Gracia-Sancho J, Lavina B, Rodriguez-Vilarrupla A, Garcia-Caldero H, Bosch J, Garcia-Pagan JC. Enhanced vasoconstrictor prostanoid production by sinusoidal endothelial cells increases portal perfusion pressure in cirrhotic rat livers. J Hepatol 2007;47:220-227.

[31] Mejias M, Garcia-Pras E, Tiani C, Miquel R, Bosch J, Fernandez M. Beneficial effects of sorafenib on splanchnic, intrahepatic, and portocollateral circulations in portal hypertensive and cirrhotic rats. Hepatology 2009;49: 1245-1256.

[32] Iwakiri Y, Groszmann RJ. Vascular endothelial dysfunction in cirrhosis. J Hepatol 2007;46:927-934.

[33] Hiukka A, Maranghi M, Matikainen N, Taskinen MR. PPARalpha: an emerging therapeutic target in diabetic microvascular damage. Nat Rev Endocrinol 2010;6:454-463.

[34] Lefebvre P, Chinetti G, Fruchart JC, Staels B. Sorting out the roles of PPAR alpha in energy metabolism and vascular homeostasis. J Clin Invest 2006; $116: 571-580$

[35] Han SH, Quon MJ, Koh KK. Beneficial vascular and metabolic effects of peroxisome proliferator-activated receptor-alpha activators. Hypertension 2005;46:1086-1092.

[36] Bosch J, Berzigotti A, Garcia-Pagan JC, Abraldes JG. The management of portal hypertension: rational basis, available treatments and future options. J Hepatol 2008;48:S68-S92.

[37] Nagula S, Jain D, Groszmann RJ, Garcia-Tsao G. Histological-hemodynamic correlation in cirrhosis-a histological classification of the severity of cirrhosis. J Hepatol 2006;44:111-117.

[38] Ogata T, Miyauchi T, Sakai S, Irukayama-Tomobe Y, Goto K, Yamaguchi I. Stimulation of peroxisome-proliferator-activated receptor alpha (PPAR alpha) attenuates cardiac fibrosis and endothelin-1 production in pressure-overloaded rat hearts. Clin Sci (Lond) 2002;103:284S-288S.

[39] Matei V, Rodriguez-Vilarrupla A, Deulofeu R, Garcia-Caldero H, Fernandez M, Bosch J, et al. Three-day tetrahydrobiopterin therapy increases in vivo hepatic NOS activity and reduces portal pressure in $\mathrm{CCl} 4$ cirrhotic rats. J Hepatol 2008;49:192-197.

[40] Morales-Ruiz M, Cejudo-Martín P, Fernandez-Varo G, Tugues S, Ros J, Angeli $P$, et al. Transduction of the liver with activated Akt normalizes portal pressure in cirrhotic rats. Gastroenterology 2003;125:522-531.

[41] Remick J, Weintraub H, Setton R, Offenbacher J, Fisher E, Schwartzbard A. Fibrate therapy: an update. Cardiol Rev 2008;16:129-141.

[42] Fernandez-Miranda C, Perez-Carreras M, Colina F, Lopez-Alonso G, Vargas C Solis-Herruzo JA. A pilot trial of fenofibrate for the treatment of nonalcoholic fatty liver disease. Dig Liver Dis 2008;40:200-205.

[43] Levy C, Peter JA, Nelson DR, Keach J, Petz J, Cabrera R, et al. Pilot study: fenofibrate for patients with primary biliary cirrhosis and an incomplete response to ursodeoxycholic acid. Aliment Pharmacol Ther 2011;33: 235-242. 